EPiC Series in Engineering
Volume 3, 2018, Pages 1911-1918
HIC 2018. 13th International
Conference on Hydroinformatics

\title{
Two-dimensional modeling of flow and contaminant transport in meandering channels
}

\author{
Il Won Seo ${ }^{1}$ and Jaehyun Shin ${ }^{2 *}$ \\ ${ }^{1}$ Seoul National University, 1 Gwanak-ro, Gwanak-gu, Seoul, 08826, South Korea \\ ${ }^{2}$ Seoul National University, 1 Gwanak-ro, Gwanak-gu, Seoul, 08826, South Korea \\ *Corresponding author: mypath80@snu.ac.kr
}

\begin{abstract}
In this study, a depth-averaged two-dimensional hydrodynamic model and scalar transport model were used to analyze the characteristics of both velocity field and concentration field of the complex flow in meandering channels. The two-dimensional shallow water model used in this study adopted the dispersion stress method in order to induce the effect of secondary flow which is normally omitted in the depth averaging process of the shallow water equation. A new vertical profile equation for the secondary flow was applied to the momentum equations by adopting the dispersion stress method. Comparisons with the experimental results of the large meandering channels of the River Experiment Center of KICT show that the computed values of the water surface profile and velocity magnitude were in good agreement with the observed data. The results of the $2 \mathrm{D}$ advection-dispersion model show that the longitudinal dispersion is much larger than theoretical results by Elder (1959) in meandering channels.
\end{abstract}

Keywords: shallow water model, advection-dispersion model, meandering channel, dispersion stress, dispersion coefficient, primary flow distribution

\section{Introduction}

Most natural rivers and channels are usually not straight due to morphology and bed topography. In order to model the flow characteristics of natural rivers, the understanding of the complex flow caused by channel meandering is necessary, but modeling river bends proves to be a challenge due to the complicated structure of the secondary flow as well as the primary flow. In curved channels, a helical flow structure is formed in the bend, which is how the secondary flow affects the flow in 
the apex. The secondary flow in meandering channels is caused by the local imbalance between the transverse water pressure forces generated by super elevation of the water surface and the vertically varying centrifugal force. The local force imbalance causes the helical flow motion to form so that the lower part of the water column flows inside, while the surface part of the water column flows outward the bend. This phenomenon causes the changes to the primary flow velocity distribution and affects the transverse dispersion rate through the mass and momentum redistribution by the helical flow structure. Thus, the understanding of the secondary flow in channel bends is necessary since it affects the primary flow distribution, channel morphology, sediment transport, and contamination movement in the area.

\section{Model development}

The derivation for the equations of motion and continuity for meandering channels were given by Rozovskii (1957), Kikkawa (1976), de Vriend (1977), etc. As an assumption for rivers, the depth was considered to be much less than the width and radius of curvature. This allowed the researcher to assume that the bank affects are minimalized in the equation derivation. They derived the vertical profile from the momentum equations which ends up in a spiral form with the upper part of the velocity profile to move outward while the lower part of the profile moved inward. Considering the former research for the vertical profiles, a developed vertical profile equation is used for the dispersion stress method.

\subsection{Flow model and dispersion stress term}

In this research using the equation for the secondary flow, the equation will be inserted into a dispersion stress form to the 2D finite element solver HDM-2D, a depth-averaged numerical model. As the meandering channel target is for rivers, the depth assumed to be smaller than the width lets the user to use a depth-averaged solver using the shallow water equation in the form of Equation (1). The HDM-2D used in this research uses the momentum equation and dispersion stress term as below (Song and Seo, 2012). Equation (2) for the dispersion stress was shown with the integration of the products of the fluctuating velocity components. It could represent the vertical variations of the velocity components in open flow situations as the original vertical variations are omitted in the depth averaging process. Also, the accurate representation of the secondary flow would be required as it would affect the transverse mixing rate significantly.

$$
\frac{\partial u_{i}}{\partial t}+u_{j} \frac{\partial u_{i}}{\partial x_{j}}=-g \frac{\partial Z}{\partial x_{i}}-g \frac{\partial H}{\partial x_{i}}+v_{T} \frac{\partial^{2} u_{i}}{\partial x_{j} \partial x_{j}}-g n^{2} \frac{u_{i} \sqrt{u_{j} u_{j}}}{h^{4 / 3}}-\frac{\partial S_{i j}}{\partial x_{j}}
$$




$$
\frac{\partial S_{i j}}{\partial x_{j}}=\frac{\partial}{\partial x_{j}}\left[h U_{i} U_{j}+\int_{h}^{H+h}\left(u_{i}(z)-U_{i}\right)\left(u_{j}(z)-U_{j}\right) d z\right]
$$

\subsection{Advection dispersion model}

A two-dimensional pollutant transport model using the advection-dispersion equation can be used for the accurate representation of mixing in the intermediate field. The two-dimensional advection-dispersion equation (2D ADE) as in Equation (3) can be derived by integrating the three-dimensional transport equation for turbulent flow over the channel depth. The 2D ADE for natural streams, in which the principle flow direction changes along the channel direction, is written in the curvilinear coordinate system as (Lee and Seo, 2010). This CTM-2D is a finite element scalar transport model, and was used to simulate the contaminant dispersion that was conducted as a tracer test in meandering channels. The model uses dispersion tensors to adequately follow the dispersion of the released tracer in the meandering channels.

$$
\frac{\partial C}{\partial t}+u \frac{\partial C}{\partial x}+v \frac{\partial C}{\partial y}=\frac{\partial}{\partial x}\left[h\left(D_{x x} \frac{\partial \bar{C}}{\partial x}+D_{x y} \frac{\partial \bar{C}}{\partial y}\right)\right]+\frac{\partial}{\partial y}\left[h\left(D_{y x} \frac{\partial \bar{C}}{\partial x}+D_{y y} \frac{\partial \bar{C}}{\partial y}\right)\right]+Q+k h \bar{C}
$$

\section{Experimental data}

The meandering channel of River Experiment Center of KICT, which is located at Andong, South Korea is a real-scale size facility in order to simulate open channel flows in a controlled outdoor environment. It has three large flumes which is capable of conducting various experiments concerning flow characteristics, river morphology and ecosystem under close-to-prototype experiment conditions. The flumes are about 11 meters wide and 600 meters long. This makes it possible to conduct various river experiments almost free from scale effects. The experiments were conducted for the comparison of the flow and dispersion of tracer in meandering channels with different sinuosities as in Fig. 1.

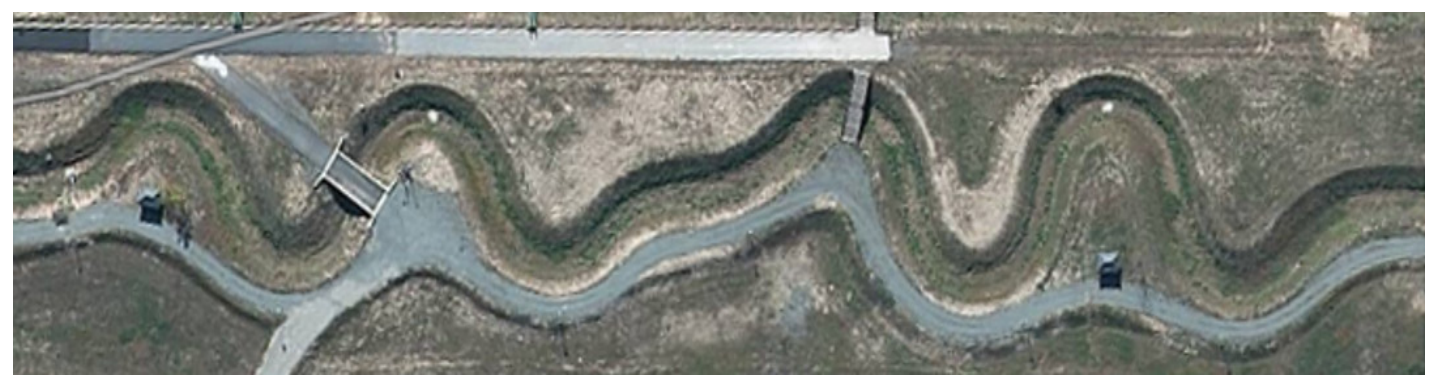

Figure 1 Aerial view of REC meandering channels 
The flow velocity measurements were conducted with an ACDP, which is the Acoustic Doppler current profiler capable of measuring water current velocities over a depth range using the Doppler effect of sound waves scattered back from particles within the water column. The tracer tests were conducted with the Rhodamine WT of $20 \%$ solute. Rhodamine WT is a fluorescence dye with reddish color that has been selected in previous tracer tests for mixing research in open channels since it is convenient to detect in low background concentrations. The tracer tests were conducted using an in situ electronic fluorometer (6130 Rhodamine WT sensor). For the injection, $150 \mathrm{~mL}$ of 20,000 ppm Rhodamine WT solution was instantaneously injected to the meandering channel. The results of the tracer test results shown in concentration-time curves are shown in Figure 2. It shows the lateral mixing occurring as the tracer passes throughout the first and second apex of the meandering channel.
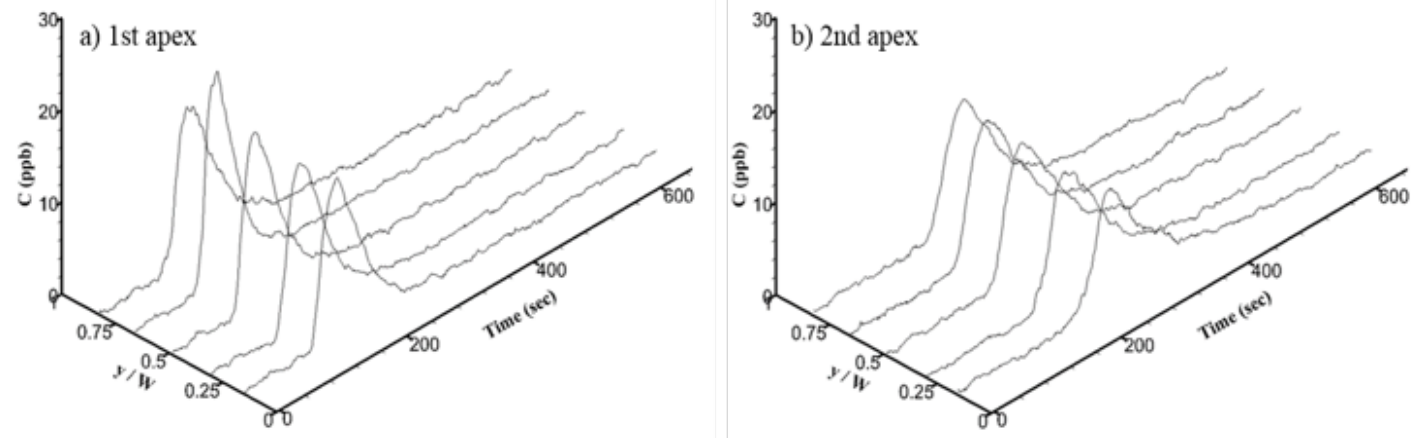

Figure 2 Tracer test results at apexes in the channel A317

The results of the experiment show that the secondary flow has affect to the primary velocity distribution in meandering channels. The peak of the velocity is located at the outer part of the bend, which is expected in sharply curved channels. It is expected that sinuosity is one of the variables that has effect to the strength of the secondary current. The velocity distribution of the channel with higher sinuosity more skewed to the outer bend than the results of low sinuosity. Also, the tracer tests show the transverse dispersion increases at the apex sections of the meandering channel, which shows the effect of the secondary flow.

\section{Numerical simulations}

\subsection{HDM-2D application}

The HDM-2D model was simulated using the dispersion stress method with the developed vertical profile equation for the secondary flow. The data comparison to 
the HDM-2D model shows good fit between the measured data from the experiment and the simulation. With the manning coefficient diversified at each sections in the meandering channels, it is could be assumed that the model produces currents in natural meandering channels quite well. The simulation model with or without the dispersion stress term was also compared to find the affects and results of the secondary flow affect to the main velocity distribution and showed the application of the dispersion stress term shows better results than the non-dispersion term results. As the maximum longitudinal velocity was located at the outer part of the apex due to the channel meander and secondary flow effects, the dispersion stress term enabled the simulated longitudinal velocity to shift toward the outer part of the apex compared to the non-dispersion stress term, to depict the secondary flow effects as in Fig. 3. This proves the dispersion stress method to be acceptable as a method for producing the effects of secondary flow to primary flow distributions.

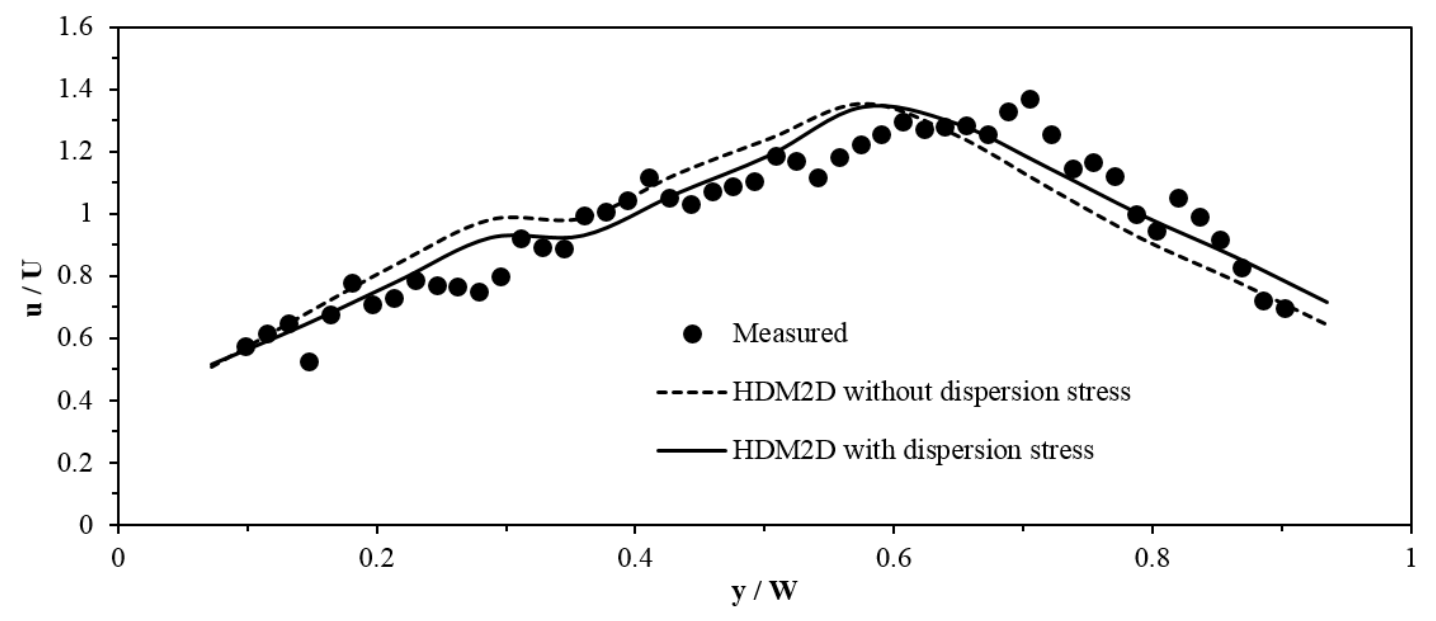

Figure 3 HDM-2D modelling results using the dispersion stress term (A315)

\subsection{CTM-2D application}

The CTM-2D model was simulated, and the results were compared with the concentration curves obtained in the tracer experiments as in Fig. 4. The timeconcentration curves of the tracer test experiments showed the skewed distribution as the secondary flow affects the transverse dispersion. The model was capable of reproducing the contaminant movement as the simulated results were in good fit with the measured data, and showed that the skewed distribution concentration curves were affected by the secondary flow. Although in the initial input phase of the tracer test there were some errors, the model showed better accuracy as the pollutant cloud passed through the meander channels and transverse mixing was undertaken. The 
comparison between the model results and tracer tests showed a good fit to prove the applicability of the 2D advection-dispersion model.

a) CTM-2D modeling
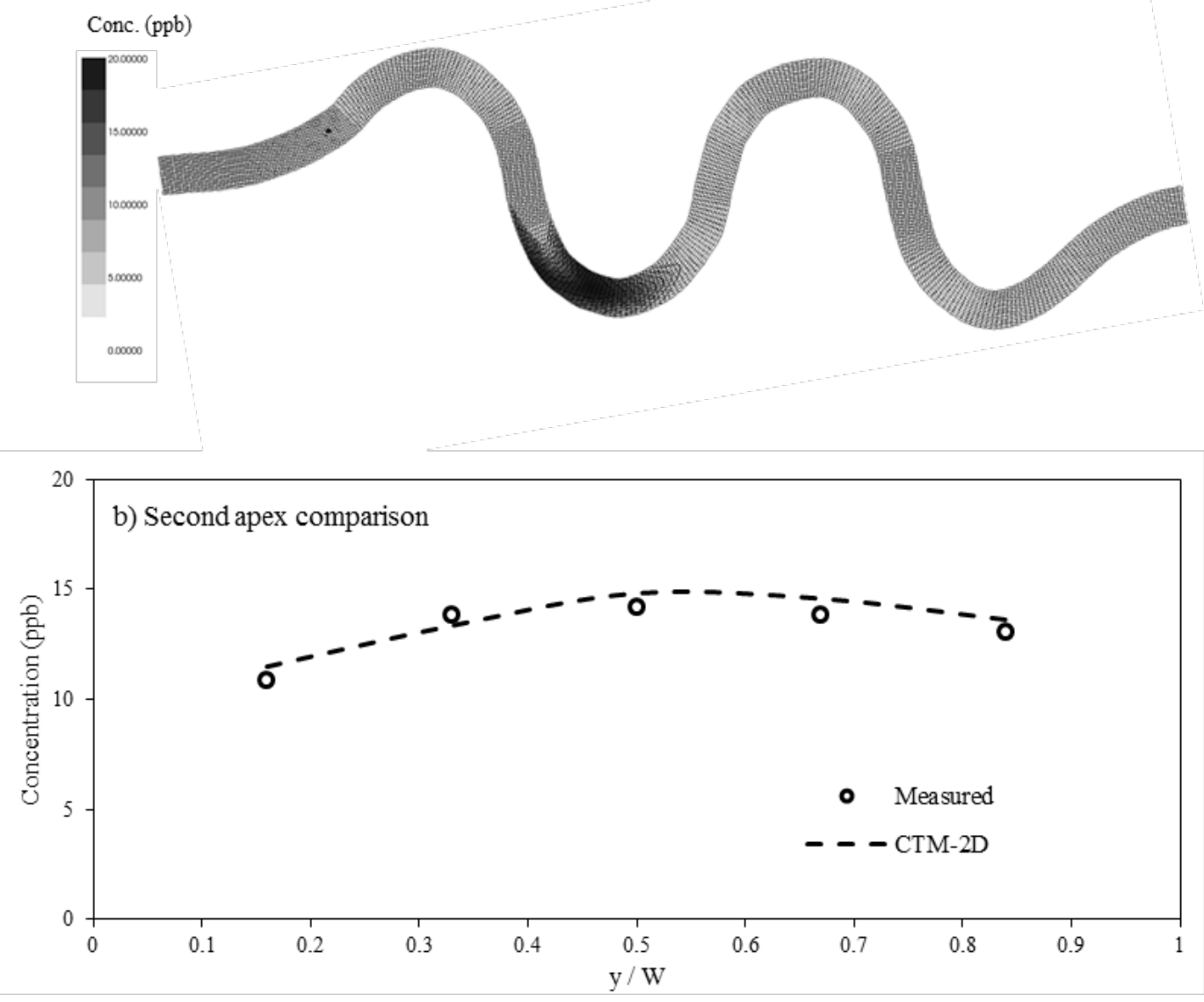

Figure 4 CTM-2D modelling results for the tracer test (A317)

The calibrated longitudinal dispersion coefficients had ranges of 10 to 15 , which was mostly larger than the 5.93 value that was suggested by Elder (1959). This is considered that the velocity deviations in meandering channels are larger than the logarithmic assumption by Elder. This is because that the Elder equation consists of the shear dispersion only while the calibrated dispersion coefficient values the effect of the irregularity of the channel and storage zones which are depicted the tail of the concentration curves. The term $D_{L S}, D_{T S}$ is the shear dispersion created by the velocity difference from the depth-averaged mean of the vertical profile such as the Elder equation value, the term $D_{L I}, D_{T I}$ is the dispersion that represents mixing by 
the irregularity of the channel and flow and the term $\varepsilon_{I}, \varepsilon_{t}$ is the mixing by turbulent motion in the two-dimensional model in Eq. 4(a) and (b).

$$
\begin{aligned}
& D_{L}=D_{L S}+D_{L I}+\varepsilon_{l} \\
& D_{T}=D_{T S}+D_{T I}+\varepsilon_{t}
\end{aligned}
$$

Since the calibrated values have to consider the $D_{L I}$ and $\varepsilon_{I}$ values, the longitudinal dispersion coefficient values results to be higher than the dispersion coefficient shown by Elder (1959).

Whereas the calibrated transverse dispersion coefficients were similar to the Fischer et al. (1979) value for meandering channel with $0.3 \sim 0.9$, as the irregularity of the channel and flow had less effect than the longitudinal dispersion coefficient values. Using the calibrated values, the mean average percent error (MAPE) of the CTM-2D model compared to the concentration curves from the tracer tests are $7.0 \%$, and $8.5 \%$ in Fig 5. (a) and (b), which shows the applicability of the CTM-2D in reproducing tracer test results.
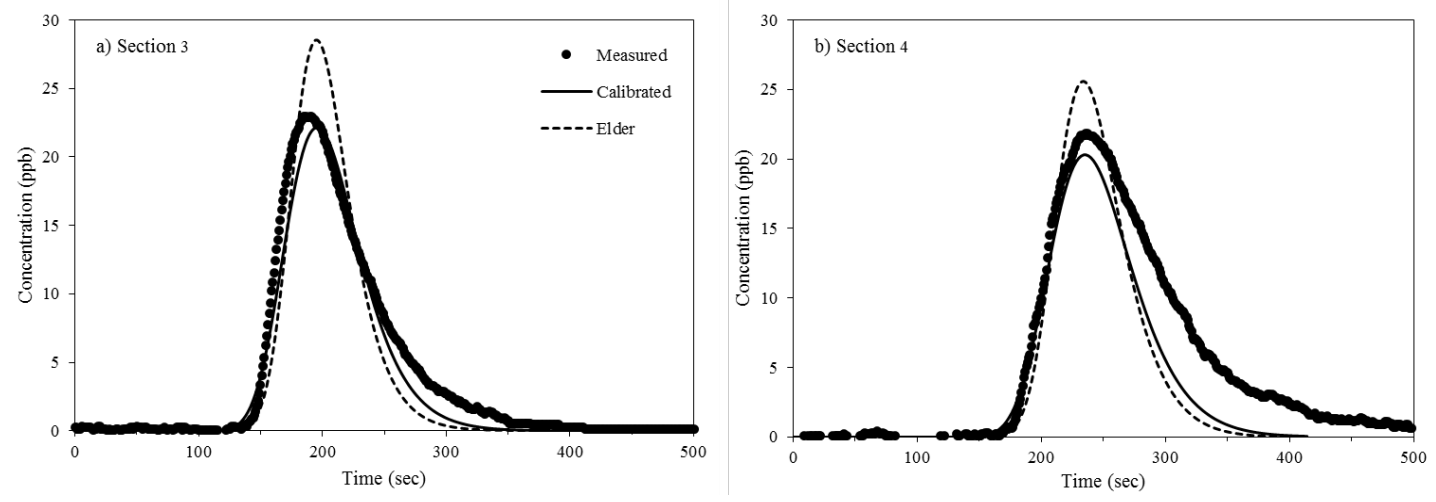

Figure 5 Concentration-time curve CTM-2D calibrated modelling results (A317)

\section{Conclusions}

The results in this paper suggest the capabilities of a depth-averaged solver with dispersion terms to reflect the effect of the secondary current in curved channels. Comparisons with the experimental results of the large meandering channels of the River Experiment Center of KICT show good agreement with the observed data. The 2D shallow water model shows some improvements compared to the solver without the secondary flow effect added in modelling open channels, especially in the regions where spiral motions in flow are significant with curvature. Modelling tracer 
tests with the 2D advection-dispersion model shows that the longitudinal dispersion is much stronger in meandering channels than theoretical results by Elder (1959). Overall, the models prove that it could reproduce the contaminant movement in meandering channels, especially the skewness of the tracer distribution as the secondary flow affects the transverse dispersion.

\section{Reference}

[1] De Vriend, H.J. (1977), "A mathematical model of steady flow in curved shallow channels." Journal of Hydraulic Research, 15, 37-54.

[2] Elder, J. W. (1959). "The dispersion of marked fluid in turbulent shear flow." J. Fluid Mech., 5, 544-560.

[3] Fischer, H. B., List, E. J., Koh, R. C. Y., Imberger, J., and Brooks, N. H. (1979). Mixing in inland and coastal waters, Academic, New York.

[4] Kikkawa, H., Ikeda, S., and Kitagawa, A. (1976), "Flow and bend topography in curved open channels," Journal of Hydraulics Engineering Division, Vol. 102, No.9, pp. 13271342.

[5] Lee, M. E., and Seo, I. W. (2010). “2D Finite Element Pollutant Transport Model for Acciddental Mass Release in Rivers," Journal of Civil Engineering, Korean Society of Civil Engineers, Springer, Vol. 14, No. 1, 2010. 1, pp.77-86.

[6] Song, C.G., Seo, I.W. (2012). "Analysis of secondary current effect in the modelling of shallow water flow in open channels." Adv. Water Resour., 41, 29-48.

[7] Rozovskii, I.L (1957), Flow of Water in Bends of Open Channels, Israel Program for Scientific Translations. 